\title{
Intra- and inter-observer variation and accuracy using different shear wave elastography methods to assess circumscribed objects - a phantom study.
}

\author{
Gregor Seliger ${ }^{1}$, Katharina Chaoui ${ }^{1}$, Christian Kunze ${ }^{2}$, Yasmina Dridi ${ }^{1}$, \\ Klaus-Vitold Jenderka ${ }^{3}$, Andreas Wienke ${ }^{4}$, Michael Tchirikov ${ }^{1}$
}

${ }^{1}$ University Clinic of Obstetrics and Prenatal Medicine, Center of Fetal Surgery, Martin Luther University Halle-Wittenberg, Halle (Saale), ${ }^{2}$ Department of Radiology, University Hospital, Halle (Saale), ${ }^{3}$ Department of Engineering and Natural Sciences, University of Applied Sciences, Merseburg, ${ }^{4}$ Institute of Medical Epidemiology, Biostatistics, and Informatics, Martin Luther University, Halle (Saale), Germany

\begin{abstract}
Aims: The elastic properties of circumscribed tissues (e.g., tendons, lymph nodes, prostates, brain tumors) are of considerable clinical interest. The purpose of this study was thus to compare the Intra-/Inter-observer variation and accuracy in vitro of point shear wave elastography (pSWE) with that of 2D-SWE and to assess 2D-SWE's precision with variable ROI (vROI) in circumscribed objects. Material and methods: Round targets (Elasticity QA Phantom Model 049) were examined for varying degrees of stiffness $(8,14,45$, and $80 \mathrm{kPa})$ and diameters $(20 / 10 \mathrm{~mm})$. Three ultrasound systems and four probes were applied (pSWE: Acuson/S3000 9L4/4C1 and Epiq7 C51, 2D-SWE: Aplio/500 PVT375BT). Three different ROIs were used, namely fixed ROI (fROI) and variable ROI: rectangular-best-fitted ROI, and round-best-fitted ROI. Each measurement was performed twice by four observers. Results: A total of 3,604 measurements were conducted. The intra-observer variation of 2D-SWE

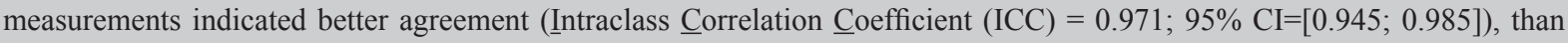
for the pSWE measurements (ICC $=0.872 ; 95 \% \mathrm{CI}=[0.794 ; 0.92])$. With both methods, the shear wave elastography applied showed low inter-observer variation: $\mathrm{ICC}=0.980 ; 95 \% \mathrm{CI}=[0.970 ; 0.987]$. However, a significant difference was observed between fROI (pSWE) and vROI (2D-SWE) on circumscribed objects in terms of accuracy. The lowest degree of observation error was detected in situations where the ROI was not "best fitted", but placed within the target of $3 \mathrm{~mm}$ from the border (target diameter: $20 \mathrm{~mm}$; mean relative error $=0.15$ ). Conclusions: When estimating the elastic properties of circumscribed tissues, the different measurement techniques performed by commercial shear wave elastography systems reveal a strong susceptibility for observational errors, depending upon the fixed $v s$. variable ROI of the pSWE $v s$. 2D-SWE technique.

Keywords: Elasticity Imaging Techniques; Acoustic Radiation Force Impulse Quantification; point shear wave Elastography; 2D shear wave Elastography; Region Of Interest
\end{abstract}

\section{Introduction}

Ultrasound (US) elastography is a method that examines tissue stiffness similarly to the human sense of

Received 15.01.2017 Accepted 22.05.2017

Med Ultrason

2017, Vol. 19, No 4, 357-365

Corresponding author: Gregor Seliger, MD

University Clinic of Obstetrics and Prenatal

Medicine,

Center of Fetal Surgery,

Martin Luther University

06120 Halle (Saale), Germany

Phone: +49 3455573921

E-mail: gregor.seliger@uk-halle.de touch. Palpation performed by physicians enables only the subjective diagnosis of tissue stiffness in regions accessible to the human hand. In contrast, US elastography yields objective (semi-)quantitative findings on tissue stiffness and also penetrates into regions of the human body previously inaccessible to physicians' hands - except intraoperatively. Other organs or tissues have thus been examined by means of US elastography besides the liver, breasts, and thyroid, for which examination techniques have already been established worldwide [1-3].

Elastic properties of circumscribed tissues and organs that are not accessible to physicians' hands (a), or which require measurements in a small, localized area (b) 
are of considerable clinical interest [1,4-17]. Examples are: tendons/ligaments ${ }^{\mathrm{a} / \mathrm{b}}$, lymph nodes ${ }^{\mathrm{a} b}$, myometria ${ }^{\mathrm{a}}$, prostates $^{\mathrm{b}}$, cervix uteri ${ }^{\mathrm{b}}$, testicles ${ }^{\mathrm{b}}$, anal sphincter muscles $^{\mathrm{b}}$, arterial plaques ${ }^{\mathrm{a} / \mathrm{b}}$, muscles ${ }^{\mathrm{a}}$, intervertebral discs ${ }^{\mathrm{a} / \mathrm{b}}$, thyroid nodules ${ }^{a / b}$, and brain tumors ${ }^{a / b}$. In such cases, it proves crucial to determine stiffness values without erroneous measurements outside the relevant organ/tissue.

Rouze et al examined circumscribed objects (spherical inclusions) with a single shear wave elastography (SWE) system using a modified Siemens SONOLINE Antares scanner (Siemens Medical Solutions USA, Inc., Ultrasound Division, Issaquah, WA, USA) [18]. The authors noted that reflections at boundaries of circumscribed objects can distort the wave shape and confound determination of the wave arrival time. Studies are needed to investigate the impact of different SWE systems and the optimal placement of the potentially variable region of interest (vROI) of measurement accuracy. This first point was investigated in several papers [19-22]. Basic phantom studies - enabling the formulation of valid statements regarding the second point - are not yet available.

Current elastography systems employ several techniques for the application of radiation force, measurement, and imaging. Strain elastography, which was not used in this study, allows (semi-) quantitative findings whereas SWE (used in this study) is quantitative. In order to obtain quantifiable results in circumscribed tissue areas, primarily two techniques were employed, namely pSWE (also referred as acoustic radiation force impulse (ARFI) quantification [16]), and bidimensional Shear Wave speed Elastography (2D-SWE). Examples of the former include Virtual Touch Tissue Quantification, VTTQ, Siemens Healthineers, Erlangen, Germany and Elast $P Q$, Philips Healthcare, Bothell, WA, USA; whilst examples of the latter comprise Shear wave elastography/Smart Maps ${ }^{\mathrm{TM}}$, Toshiba Medical Systems, Minato, Japan; ShearWave TM Elastography/Supersonic shear imaging (SSI), SuperSonic Imagine, SA, Aix-en-Provence, France; LOGIQ E9 2D Shear Wave Elastography, GE Healthcare, Wauwatosa, WI, USA [16,23,24].

The point SWE (pSWE), which enable measuring the speed of a shear wave via tissue displacement within a ROI caused by the passing shear wave, provide a quantitative measure of shear wave speed $\mathrm{c}_{\mathrm{s}}$, which is linked to tissue stiffness and focused on the fixed ROI (fROI), with a predefined size, set by means of the system (VTTQ/ ElastPQ: fROI box with predefined sizes of $10 \times 5 \mathrm{~mm}^{2}$ (VTTQ), $5 \times 5 \mathrm{~mm}^{2}$ (VTTQ, fig 1a), and $13 \times 10 \mathrm{~mm}^{2}$ (ElastPQ, fig 1b), respectively.

The SWE technique, also called shear wave speed imaging (SWSI) or shear wave elasticity imaging (SWEI), relies on ARFI and detection of shear waves with high pulse or image repetition frequencies and subsequent determination of the shear wave speed. With products like Shear wave elastography/Smart Maps ${ }^{\mathrm{TM}}$ (Toshiba Medical Systems, Minato, Japan) the shear wave velocities generated by ultrasound acoustic push pulses can be measured inside a bidimensional box/area (several $\mathrm{cm}$ per side) instead at a single point, producing a so-called bidimensional Shear Wave speed Elastography (2D-SWE). Freezing images can subsequently be viewed using three different display modes, namely Propagation (arrival time contour) mode (fig 1c), Speed (shear velocity) mode (m/s) (fig 1d), and Elasticity mode $(\mathrm{kPa})$. Elastic properties of the circumscribed tissue are measured by placing a vROI inside the sample box. This 2D averaged area should overcome the limitation of conventional pSWE to inadvertently investigate only small regions of circumscribed objects. The shear wave quality indicator - Propagation (arrival time contour) mode (feature only available with the product of Toshiba Medical Systems, Minato, Japan) - should provide real-time feedback and optimize placement of the vROI - which still awaits validation. Quantitative information of tissue stiffness can be obtained in this shear wave speed imaging by captur-

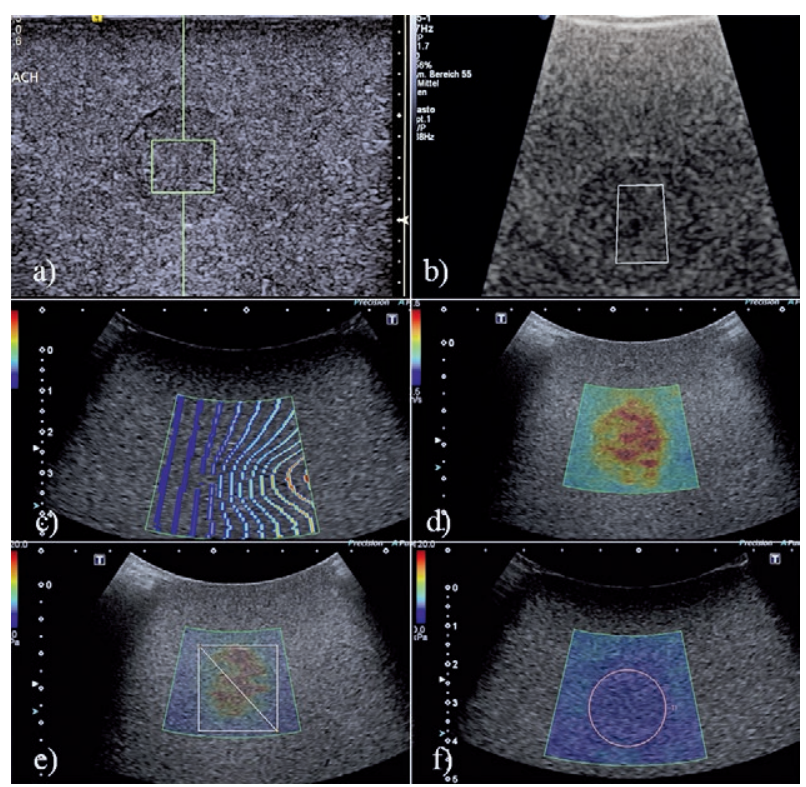

Fig 1. Shear wave elastography methods: a) point Shear Wave Elastography (pSWE); fixed Region Of Interest (ROI) / ROI set at the center of a target; Acuson3000 / 4C1 Convex-probe (Method 1); b) pSWE, fixed ROI / ROI set at the center of a target; Epiq7 / C5-1 Convex-probe (Method 3); c) bidimensional Shear-Wave-Elastography (2D-SWE); Aplio 500 / 375BT Convex-probe; Propagation-Mode (arrival time contour); d) 2DSWE; Aplio 500 / 375BT Convex-probe - Speed (shear velocity) mode; e) 2D-SWE; variable ROI / rectangular-best-fitted; Aplio 500 / 375BT Convex-probe (Method 4); f) 2D-SWE; variable ROI / round-best-fitted; Aplio 500 / 375BT Convexprobe (Method 5) 
ing the transverse shear waves propagation induced by the displacement of tissues under the excitation of ultrasound acoustic push pulses [25]. Tissue excitation in 2D-SWE is similar to that in pSWE, the wave speed is calculated using wave-peak detection and a time-of-flight algorithm.

The question as to whether pSWE with either fixed ROI or 2D-SWE with variable ROI exhibited greater accuracy in determining the elasticity of circumscribed objects remains unanswered thus far.

The aim of this study was thus to compare the inter-observer variation, the intra-observer variation, and the test accuracy of point SWE with that of 2D-SWE in circumscribed objects in vitro and to assess 2D-SWE's precision with variable ROI in circumscribed objects in vitro using a phantom training model with multiple operators on three commercially available SWE devices, widely used in clinical practice. Our underlying aim was to evaluate the different technologies in terms of variation and accuracy to examine the elastic properties of circumscribed objects.

\section{Material and methods}

\section{Study design - general information}

This investigation is a phantom study involving eight elasticity phantom targets, four investigators (with varying experience levels), three US systems, and four probes over two days, with a one-week interval between measurement sessions.

\section{Phantom}

For the study, we utilized a commercially available Elasticity QA Phantom Model 049 (Computerized Imag- ing Reference Systems Company Inc., Norfolk, Virginia, USA). The phantom contained targets for known stiffness. Eight spheres with 2 and $1 \mathrm{~cm}(4$ each) diameters and four different stiffness's, namely $80,45,14$, and 8 $\mathrm{kPa}$, were embedded (background medium stiffness of $25 \mathrm{kPa}$ through use of Zerdine [26]). Consequently, there were two spheres that were softer than the background, while the other two were harder. The spheres were placed in two layers, with the cylinders' centers at depths of 1.5 (A) and 3.0 (B) cm, respectively (fig 2). Background and targets material was made of Zerdine ${ }^{\circledR}$ (Speed of Sound: $1540 \mathrm{~m} / \mathrm{s}$; Attenuation: $0.50 \mathrm{~dB} / \mathrm{cm}-\mathrm{MHz}$ ).

\section{Ultrasonography (pSWE and 2D-SWE)}

US examinations were performed using for pSWE: Acuson S3000 1.5: linear 9L4 and convex 4C1 US probe (Software Syngo VE31F SL04P09, version 400.1.016; Siemens Healthineers, Erlangen, Germany) and Epiq 7G 1.5 convex C5-1 US probe (software version 1.3.2; Philips Healthcare, Bothell, WA, USA) and for 2D-SWE:

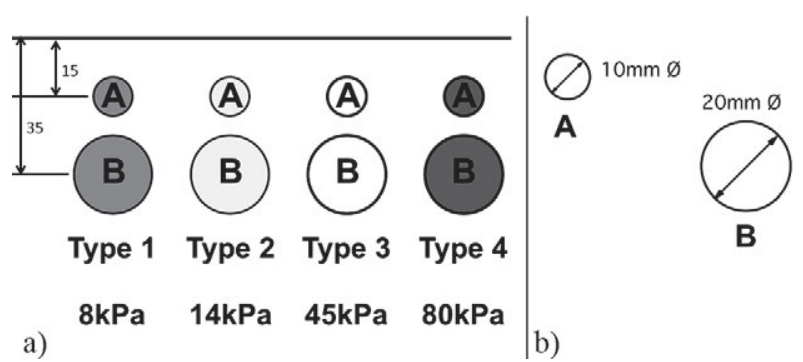

Fig 2. Elasticity QA Phantom Model 049 (Computerized Imaging Reference Systems Company Inc., Norfolk, Virginia, USA): a) front view, targets: 8 spheres with 2 and $1 \mathrm{~cm}$ diameters and four different stiffness's; b) right end view.

Table I. Methods of shear wave elastography.

\begin{tabular}{|c|c|c|c|c|c|c|c|c|c|c|c|}
\hline \multirow{2}{*}{\multicolumn{2}{|c|}{$\begin{array}{l}\text { Method (M) } \\
\text { of elastogra- } \\
\text { phy }\end{array}$}} & \multirow[t]{2}{*}{ ROI } & \multirow[t]{2}{*}{ Machine } & \multirow{2}{*}{$\begin{array}{l}\text { Probe / } \\
\text { Transducer }\end{array}$} & \multicolumn{7}{|c|}{ Default settings } \\
\hline & & & & & \multirow{2}{*}{$\begin{array}{l}\text { Frequen- } \\
\text { cy Band- } \\
\text { width } \\
4.0-9.0 \\
\mathrm{MHz}\end{array}$} & \multirow{2}{*}{$\begin{array}{l}\text { Maximum } \\
\text { Field of } \\
\text { View } \\
147 \mathrm{~mm}\end{array}$} & \multirow{2}{*}{$\begin{array}{l}\begin{array}{l}\text { Maximum } \\
\text { Display } \\
\text { Depth }\end{array} \\
140 \mathrm{~mm} \\
\end{array}$} & \multirow{2}{*}{$\begin{array}{l}\begin{array}{l}\text { Number } \\
\text { of Ele- } \\
\text { ments }\end{array} \\
576\end{array}$} & \multicolumn{2}{|c|}{ Footprint } & \multirow{2}{*}{\begin{tabular}{|l|}
$\begin{array}{l}\text { Max. } \\
\text { index } \\
\text { value }\end{array}$ \\
1.70 \\
\end{tabular}} \\
\hline M1 & pSWE & fROI\# & $\begin{array}{l}\text { Acuson } \\
\text { S3000 } 1.5\end{array}$ & $\begin{array}{l}\text { 9L4 Linear- } \\
\text { probe }\end{array}$ & & & & & $40 \mathrm{~mm}$ & $\begin{array}{l}\text { 2D Steer- } \\
\text { ing Angle: } \\
\max . \pm 20^{\circ}\end{array}$ & \\
\hline M2 & pSWE & fROI\# & $\begin{array}{l}\text { Acuson } \\
\text { S3000 } 1.5\end{array}$ & $\begin{array}{l}4 \mathrm{C} 1 \text { Convex- } \\
\text { probe }\end{array}$ & $\begin{array}{l}1.0-4.5 \\
\mathrm{MHz}\end{array}$ & $70^{\circ}$ & $300 \mathrm{~mm}$ & 128 & $61 \mathrm{~mm}$ & - & 1.88 \\
\hline M3 & pSWE & fROI\# & Epiq7 1.5 & $\begin{array}{l}\text { C5-1 Con- } \\
\text { vex-probe }\end{array}$ & $\begin{array}{l}1.0-5.0 \\
\mathrm{MHz}\end{array}$ & $111^{\circ}$ & $300 \mathrm{~mm}$ & 160 & $45 \mathrm{~mm}$ & - & 1.30 \\
\hline M4 & 2D-SWE & $\begin{array}{l}\text { vROI*- } \\
\text { rectan- } \\
\text { gular }\end{array}$ & $\begin{array}{l}\text { Aplio } 500 \\
\text { V } 6.0\end{array}$ & $\begin{array}{l}375 \mathrm{BT} \\
\text { Convex- } \\
\text { probe }\end{array}$ & $\begin{array}{l}1.0-6.0 \\
\mathrm{MHz}\end{array}$ & $70^{\circ}$ & $250 \mathrm{~mm}$ & 192 & $72 \mathrm{~mm}$ & $\begin{array}{l}\text { Scan } \\
\text { Range: } \\
\max .50 \%\end{array}$ & 1.50 \\
\hline M5 & 2D-SWE & $\begin{array}{l}\text { vROI*_ } \\
\text { round }\end{array}$ & $\begin{array}{l}\text { Aplio } 500 \\
\text { V } 6.0\end{array}$ & $\begin{array}{l}375 \mathrm{BT} \\
\text { Convex- } \\
\text { probe }\end{array}$ & $\begin{array}{l}1.0-6.0 \\
\mathrm{MHz}\end{array}$ & $70^{\circ}$ & $250 \mathrm{~mm}$ & 192 & $72 \mathrm{~mm}$ & $\begin{array}{l}\text { Scan } \\
\text { Range: } \\
\max .50 \%\end{array}$ & 1.50 \\
\hline
\end{tabular}

pSWE - point Shear Wave Elastography; 2D SWE - bidimensional Shear-Wave-Elastography; f or v ROI - fixed or variable Region-OfInterest / \#ROI set at the center of a target / *best fitted 
Aplio 500 convex PVT 375BT US probe (software version 6.0; Toshiba Medical Systems, Minato, Japan) (table I). All indicated elasticity values are given as Young's moduli.

Acuson S3000 and Epiq7 were employed in order to evaluate pSWE by placing the fixed ROI in the center of target (10x5 $\mathrm{mm}^{2}$ and $5 \times 5 \mathrm{~mm}^{2}$ (VTTQ), and $13 \times 10 \mathrm{~mm}^{2}$ (ElastPQ). Aplio 500 was used to assess 2D-SWE by placing the rectangular-variable or round-variable ROI best fitted around the round target. The ROI was placed by the examiner exactly in the center of the phantom target. Therefore the depth from the phantom surface to center of the ROI was the same in the same measuring series. The depth of the target was known (according to the manufacturer)

An indoor, air-conditioned room was used; standardized conditions (constant air humidity, constant room temperature, and same lighting conditions) were ensured.

\section{Study design - measurement procedures}

For the first part of study (I) - verification of inter/ intra-observer variation (Ia) and test accuracy (Ib) when considering the utilization of pSWE vs. 2D-SWE on circumscribed objects - we employed five measurement methods detailed in Table I.

Each measurement session was performed twice (day 1 and day 8) by four observers. Observers 1, 2, 3, and 4 had US experience levels (exp.lev.) of 25, 25, 15, and 5 years, respectively and $3,2,1$, and 0 years of experience with US SWE, respectively. The measurement was repeated 10 times. Stiffness values were defined as the median of these 10 measurements for a single target.

We measured the SWV (m/s) / Young's modulus $E$ $(\mathrm{kPa})$ for each individual sphere in terms of verification of inter/intra-observer variation (Study part Ia) for Types 1-4/B and Type 4/A with all probes, and Types 1-3/A with only Acuson S3000/9L4 linear probe (method 1), as the target was clearly visible with this probe alone. For test accuracy verification (Study part Ib), measurements were taken for Types 1-4/B and Type 4/A with all probes in order to ensure the inter-comparability of methodologies.

For the second part of study (II) - verification of test accuracy when considering the utilization of the vROI on circumscribed objects - we employed an adapted variant of the measurement method 5 (2D-SWE, round-variable ROI, Aplio 500, 375BT convex probe). This measurement session was performed by a single operator. He measured the $\mathrm{SWV}(\mathrm{m} / \mathrm{s}) /$ Young's modulus $E(\mathrm{kPa})$, respectively, of the spheres/targets for Types 1-4/B with 2D-SWE, Aplio 500, 375BT convex probe and round-variable ROI. More specifically, in order to investigate the impact of the vROI's dimensions in relation to the target dimension on the accuracy of the measurement results, we performed

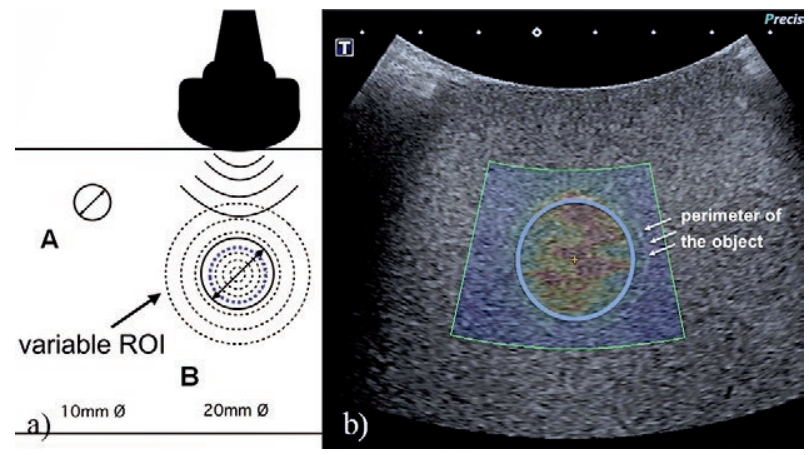

Fig 3. Method second part of study (II): a) Elasticity QA Phantom Model 049 (Computerized Imaging Reference Systems Company Inc., Norfolk, Virginia, USA) - with Convex-probe: 2D-SWE; variable round ROI; Aplio 500 / 375BT Convexprobe: b) 2D-SWE; round-variable ROI / ROI not best fitted placed within the target $3 \mathrm{~mm}$ from the border; Aplio $500 /$ 375BT Convex-probe

twenty measurements using different diameters (from 40 $\mathrm{mm}$ to $2 \mathrm{~mm}$ ) for each phantom target. The center of the ROI was congruent with the center of the phantom target. During the measurement with a $20 \mathrm{~mm}$ ROI, its border was congruent with the target border (fig 3 ). The relative error is the absolute error divided by the magnitude of the exact value (manufacturer's data of the phantom lesions). The absolute error is the magnitude of the difference between the exact value and the measurement variable obtained as the median of 10 single measurements.

In the first part of the study - Method 5 (2D-SWE, Aplio 500, variable-round ROI, best fitted) showed low variability and no outliers. Hence, the measurement procedure in the second part of the study was repeated five times for each target type, with the stiffness value defined as the mean of the five measurements for each ROI diameter (diameters: 40, 38, 36, 34, 32, 30, 28, 26, 24, 22, 20, $18,16,14,12,10,8,6,4$ and $2 \mathrm{~mm}$ ).

\section{Statistical analysis}

Statistical analyses were performed using SPSS software (Version 21, IBM, Armonk, NY, USA). Intra-observer variation denotes the degree of agreement among repeated measurements performed by the same observers. This was analyzed by Bland-Altman-plots [27] and by the Intraclass Correlation Coefficient (ICC) [28] between the (medians of the 10) measurements of day 1 and day 8. Inter-observer variation means the degree to which two or more independent observers measuring the same objects agree on what they observe. This was measured by ICC between the (medians of the 10) measurements of all observers and exemplary analyzed by Bland-Altman-plots. Measurement accuracy in vitro denotes the extent to which a measurement accurately measures the true value. This is analyzed in the form of boxplots of the 


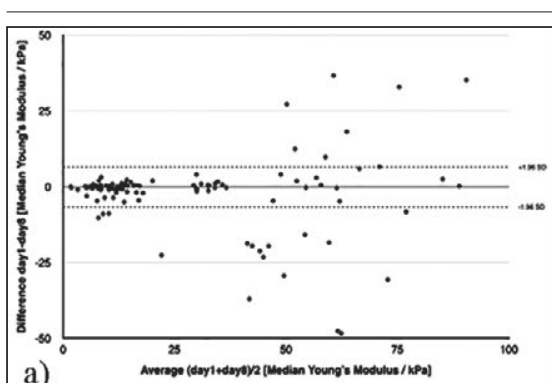

a)
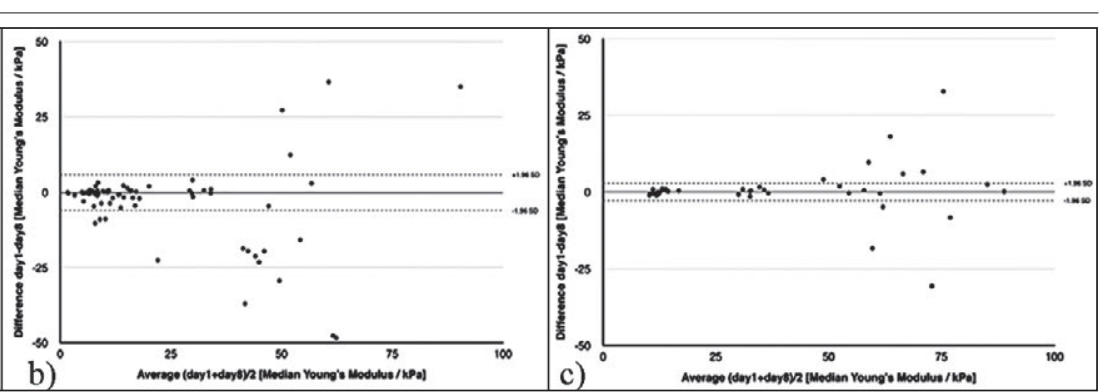

Fig 4. Intra-observer variation. Bland Altman plots (Difference against mean) showing agreement in Median Young's (Elastic) Modulus (E) $[\mathrm{kPa}]$ (Median of the 10 measurements of one target) for all observers and all targets between day 1 and day 8 . Solid line represents the mean difference between days and dotted lines represents mean $+/-1.96$ standard deviations $(\mathrm{SD})$. $\mathrm{pSWE}=$ point Shear Wave Elastography. 2D-SWE = bidimensional Shear-Wave-Elastography: a) all methods combined $(\mathrm{n}=112)$; $b$ ) methods 1-3 (pSWE) $(\mathrm{n}=72) ; \mathrm{c})$ methods 4 and $5(2 \mathrm{D}-\mathrm{SWE})(\mathrm{n}=40)$

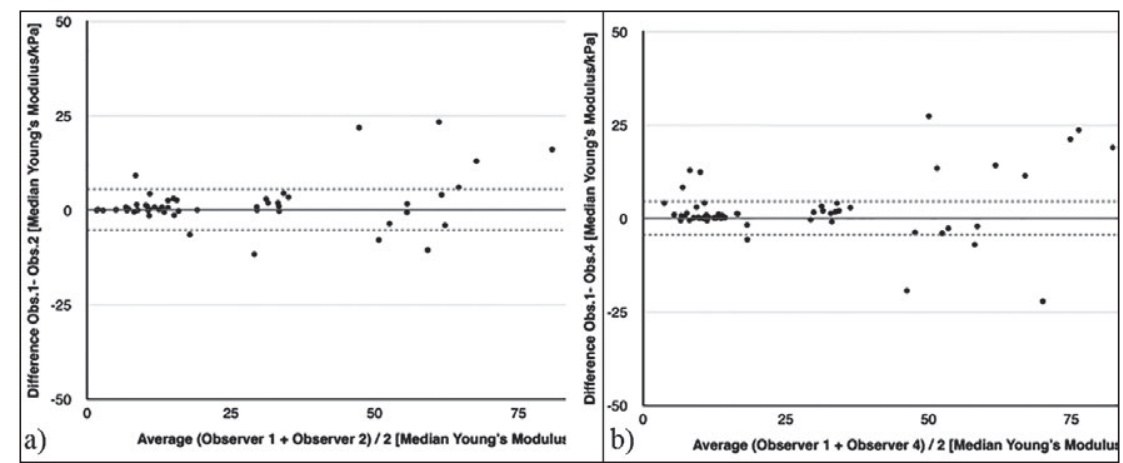

Fig 5. Inter-observer variation. Bland Altman plots (Difference against mean) showing agreement in Median Young's (Elastic) Modulus (E) [kPa] (Median of the 10 measurements of one target) for all methods, all days and all targets between observers. Solid line represents the mean difference between observers and dotted lines represents mean + 1.96 standard deviations (SD). Obs. = Observer. US experience levels = exp.lev. and years $=$ y. : a) observer 1 (exp.lev.25y) versus Observer 2 (exp.lev.25y) $(n=56)$; b) observer 1 (exp.lev.25y) versus Observer 4 (exp.lev. 5y) $(n=56)$.

relative difference of the (medians of the 10) measurements from the true value of the phantom.

\section{Results}

Overall 3,604 measurements were conducted under standardized conditions.

Study part I: verification of intra/inter-observer variation (Ia) and test accuracy (Ib) using different measurement methods

\section{Intra-observer variation}

Overall the intra-observer variation of Young's modulus (E) $[\mathrm{kPa}]$ measurements showed a ICC value of 0.929 $(95 \% \mathrm{CI}[0.897 ; 0.951])$. The intra-observer variation for methods 1-3 (pSWE) had an ICC value of $0.872(95 \%$ CI $[0.794 ; 0.92])$. The intra-observer variation of Young's modulus $(E)[\mathrm{kPa}]$ 2D-SWE measurements indicated better agreement, namely a ICC value of 0.971 (95\% CI $[0.945 ; 0.985]$ ), than for the pSWE measurements (fig 4).

\section{Inter-observer variation}

Inter-observer variation of all operators combined (all targets and for $\mathrm{pSWE} / 2 \mathrm{D}-\mathrm{SWE}$ combined) resulted in an ICC value of 0.98 (95\% CI [0.970; 0.987]). The interobserver variation as outcomes of observer $1 v s$. observer 2 was $0.949(95 \%$ CI $[0.911 ; 0.970])$ and as outcomes of observer 1 vs. observer 40.962 (95\% CI [0.934; 0.978]) (fig 5).

\section{Accuracy}

The overall accuracy of results proved to be nearly independent of observer experience levels. The results are displayed in figure 6 and 7. Considerable variability was observed for stiffness value measurements of $80 \mathrm{kPa}$ targets, irrespective of operators' level of experience.

A significant difference between the pSWE with fixed ROI vs. 2D-SWE with variable ROI on circumscribed objects was found in terms of accuracy (fig 8). Method 3 exhibited the greatest variability of measured stiffness values (median of the 10 measurements for one target). 


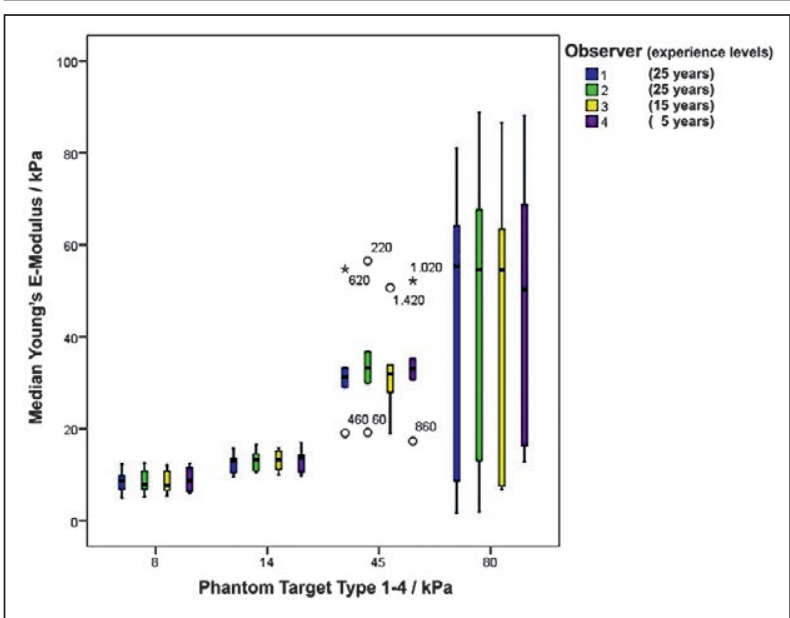

Fig 6. Phantom Target Type versus Mean Young's E-Modulus for Observer 1-4.Boxplots of the absolute values of stiffness in Median Young's (Elastic) Modulus (E) [kPa] (Median of the 10 measurements of one target) showing accuracy between observers for Phantom Target Types 1-4 (for all methods and all days combined). US Experience level is specified. $n=224$.

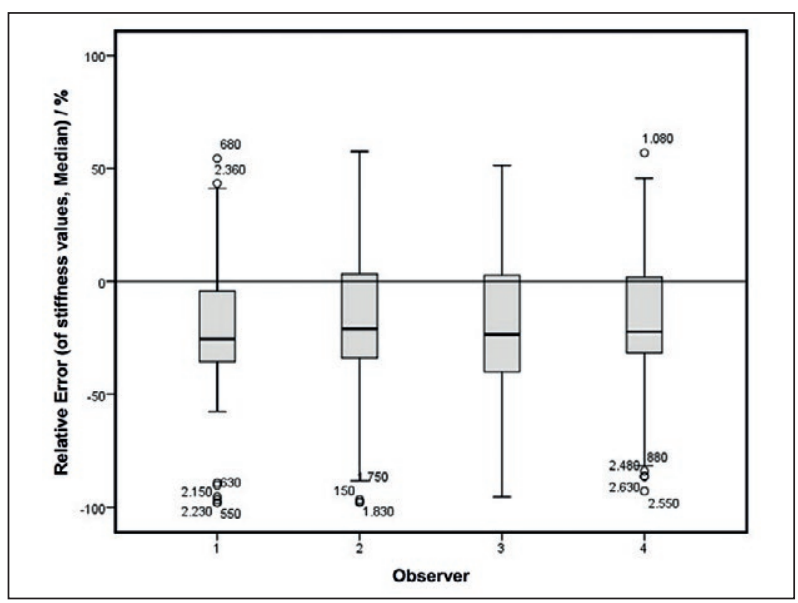

Fig 7. Observer versus Relative Error of stiffness values. Boxplots of the relative difference of the (medians of the 10) measurements from the true value of the phantom in \% showing accuracy for observers (for all Phantom Target, all methods and all days combined). Observers $1,2,3$, and 4 with US experience levels $25,25,15$, and 5 years, respectively. $n=224$.

However, relative error seemed to be dependent upon the 2D-SWE (variable ROI / rectangular-best-fitted = methods 4) of surrounding tissues (background medium stiffness $25 \mathrm{kPa}$ ) when measured whilst employing a "best fitted" ROI (fig 9). If the stiffness of the surrounding tissue (background medium) is greater than the stiffness of the target, then the stiffness of the target is overestimated. If the stiffness of the surrounding tissue is less than the stiffness of the target, then the stiffness of the target is underestimated.

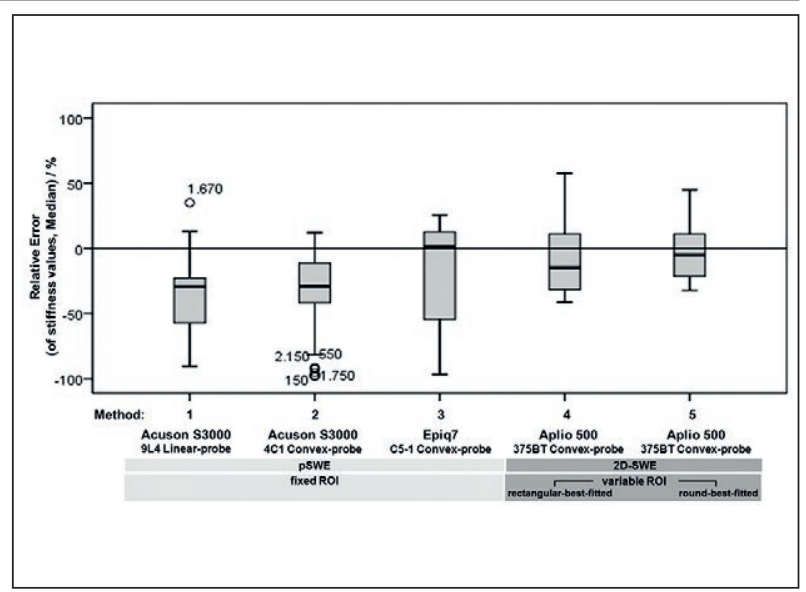

Fig 8. Methods of elastography versus Relative Error of stiffness values. Boxplots of the relative difference of the (medians of the 10) measurements from the true value of the phantom in $\%$ showing accuracy for methods 1-5 for all Phantom Target, all observers, and all days combined. Mann-Whitney U test: significant difference between Method 1, 2, 3 (pSWE) versus Method 5, 6 (2D-SWE) $(\mathrm{p}<0.001) . \mathrm{n}=224$.

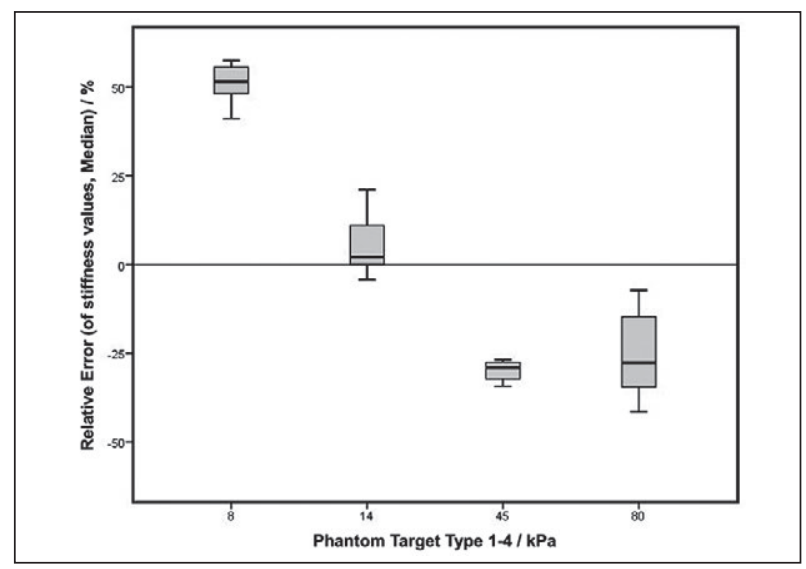

Fig 9. Method 4: Phantom Target Type versus Relative Error of stiffness values. Boxplots of the relative difference of the (medians of the 10) measurements from the true value of the phantom in \% showing accuracy for method 4 (2D-SWE; variable ROI / rectangular-best-fitted; Aplio 500 / 375BT Convexprobe) for all observers and all days combined. Background medium stiffness $25 \mathrm{kPa}$. $\mathrm{n}=40$.

Study part II: verification of test accuracy whilst considering application of the variable round ROI (2DSWE) on circumscribed round objects

The least relative measurement error was detected in situations where the ROI was not "best fitted" but placed within the target of $3 \mathrm{~mm}$ from the limit (relative error $[\delta]$ $=0.15$; error percent $[\delta]=15 \%$; target diameter $=20 \mathrm{~mm}$ ) (fig 10). The relative error is more than halved by placing the ROI within the target of $3 \mathrm{~mm}$ from the limit instead of placing $3 \mathrm{~mm}$ outside the object boundary. 


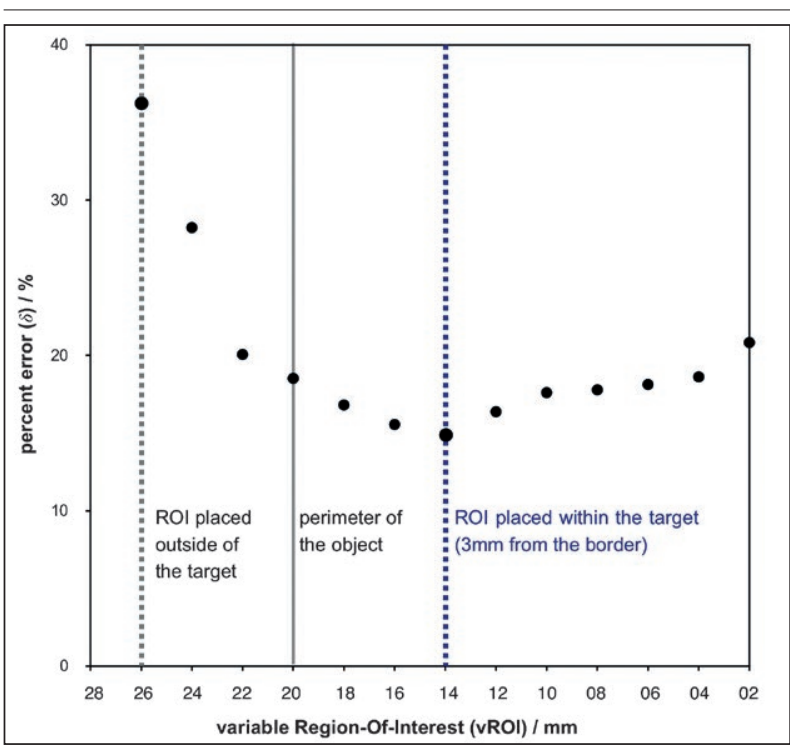

Fig 10. 2D-SWE with round-variable ROI on circumscribed round objects. Percent error as a function of different ROI size in mm. Method: Elasticity QA Phantom Model 049 (Computerized Imaging Reference Systems Company Inc., Norfolk, Virginia, USA) - with Convex-probe: 2D-SWE; round-variable ROI; Aplio 500 / 375BT (blue line = ROI (not best fitted!) placed within the target $3 \mathrm{~mm}$ from the border). $\mathrm{n}=80$.

\section{Discussions}

US elastography is increasingly used for the diagnosis of stiffness in numerous circumscribed tissues [1,417]. To date, there has been a lack of phantom studies which have systematically assessed the current US shear wave elastography techniques (pSWE with fixed ROI vs. 2D-SWE with variable ROI) in an effort to evaluate their suitability/test performance for this condition.

Three out of four operators in a phantom study by Mun et al demonstrated "good intra-observer variation" in real-time 2D-SWE quantitative elastography assessment (Supersonic shear imaging $(\mathrm{SSI})^{\mathrm{TM}}$, Aixplorer ultrasound system, SuperSonic Imagine, Aix-en-Provence, France) [29]. Zhang et al [15] demonstrated a correlation coefficients of $0.904, p<0.001$ for intra-observer measurement concerning pSWE when repeated measurements were performed by the same operator for the same nodule in 30 patients within only two days [15]. In our study, intra-observer variation for 2D-SWE indicated a better agreement than for pSWE.

Quantitative US elastography (pSWE and 2D-SWE) was highly reproducible with good agreement across observers, constituting an advantage of the elastography methods - pSWE and 2D-SWE. Data regarding other settings has been presented by Mun et al and Dillman et al $[19,29]$. Their data also confirmed "good test per- formance" of such US SWE techniques (SWE/ARFI) in terms of inter-observer variation.

Observers' experience levels had little impact upon the accuracy of measurement results in the phantom experiment. The considerable variability of stiffness value measurements of $80 \mathrm{kPa}$ targets was previously reported and proved to be independent of operators' experience levels $[20,30,31]$.

We confirm exploratory analyses from other authors [20] claiming that the pSWE method exhibits problems when performing valid target measurements with high stiffness values such as 45 and $80 \mathrm{kPa}$. In the case of the $45 / 80 \mathrm{kPa}$ phantom targets, the imaging algorithm of the systems is not optimized, which causes errors in measurements. At $45 / 80 \mathrm{kPa}$, the shear wave speed may have been too fast and the displacements too small, so that the systems, due to limited frame rates and resolution, were not able to detect the shear wave appropriately (the frame rate will also be affected by the maximum imaging depth) [32]. Furthermore, the method "time-to-peak" (TTP; used by PHILIPS Epiq7 and SIEMENS Acuson S3000 - pSWE), which relies on the identification of the peak displacement, is sensitive to changes in wave shape introduced by reflections at boundaries of the spherical targets and especially at boundaries with high differences of the E-Modulus [18]. The "time-to-peak-slope" (TTPS) method determine the wave arrival time by estimating the position of the leading edge of the wave. Since this edge precedes the peak displacement, the arrival time occurs before the time of peak displacement. There is less opportunity for the reflection from the boundaries of spherical inclusion to sum constructively at the leading edge of the wave to skew the arrival times [18]. In addition, errors introduced by these reflections increase with the increasing shear wave length. This indicates a larger error at high shear wave velocities.

Pfeifer et al revealed deviating pSWE values, as assessed by means of 6C1HD and 4C1 transducers (Acuson S2000; Siemens Healthineers, Erlangen, Germany) [21]. They postulate that the difference between the probes might be due to the fact that the number of elements and channels differs between both transducers and that the beam profile is shaped slightly different over depth. Shin et al showed that differences between pSWE $v s$. Supersonic shear imaging (SSI) ${ }^{\mathrm{TM}}$ values (Aixplorer ultrasound system, SuperSonic Imagine, Aix-en-Provence, France) depended not only on the transducers but also on US machines from different vendors (e.g., with different F-number and the frame rate of imaging) [22].

pSWE values assessed in our elasticity phantom study with the Method 3 (Epiq7, C5-1 convex probe) were slightly higher than those recorded with Methods 1 and 2 
(Acuson $\mathrm{S} 3000$, 9L4 linear probe and 4C1 convex probe. However, the accuracy of diagnostic tests was quite similar between both US devices (Epiq7, Acuson S3000), with only small differences between the two US devices.

Contrary to findings by Shin et al while comparing the measurement of Methods 1 and 2 (Acuson S3000, 9L4 linear probe and 4C1 convex probe), we observed no differences in the mean relative error [22]. The following ultrasonic probes were not available for the presented study: Acuson S3000 - 6C1HD, 4V1 and Aplio 500: PLT 1005BT. (Philips does not offer any other probe for pSWE.) Future studies with these transducers are needed to verify our results.

The question as to whether pSWE with either fixed ROI (Methods 1, 2, and 3) or 2D-SWE with variable ROI (Methods 4 and 5) exhibited greater accuracy in determining the elasticity of circumscribed objects remains unanswered thus far. The 2D-SWE technique by medians of vROI promises enhanced accuracy while measuring elasticity of circumscribed objects. Considerable inaccuracies have been detected in previous studies - particularly when the fROI was larger than the measured object [20]. Carlsen et al suggest that "Including parts of the surrounding medium could account this effect on elastographic measurements" of circumscribed objects. We can confirm this effect.

From the variable ROI (vROI), it is hoped to obtain valid values by adjusting the ROI in object form. For the first time, the dependency of the test accuracy when considering the application of a variable ROI (2D-SWE) on circumscribed objects has been verified. The least relative measurement error was observed in situations where the ROI is not "best fitted", but placed within the target. Havre et al already described in their strain elastography study that placing the reference ROI area closer to the source of stress yields strain ratio values with greater intra-observer variability than any of the other scanning setups [33]. Fukuhara et al acknowledge in their study that when objects exhibit small diameters, measurements of SWV parameters are unstable. They interpret the phenomena of this depreciation as being accounted for by the US waves of the push pulse, which were reflected and refracted at the boundaries of the objects, thus resulting in the irregular production of shear waves $[15,34]$. Such factors in the border area of the phantom object may be responsible for the high relative error of US shear wave elastography measurements when adapting the ROI directly to the shape of the object ("best fitted").

Finally, the most valid method for detecting stiffness of circumscribed (phantom) objects in our study was 2D-SWE with variable ROI. It implies that caution is required when comparing results not only obtained by means of transducers, but also between different shear wave elastography machines and techniques employed in clinical practice.

\section{Limitation of the study}

Due to our use of an elasticity phantom, any results must be verified in vivo. Owing to its nature as a phantom study, we were unable to simulate the various clinical conditions of patients which may have influenced results. In particular, the irregular border of natural measured objects may have constituted another source of error. The freehand definition of ROI is possible but must undergo additional verification. The comparison of accuracy between the fixed ROI $v s$. variable ROI is limited by the fact that fixed ROI and variable ROI was not used in the same imaging systems.

\section{Conclusions}

SWE is an established method subject to widespread use, e.g., for the assessment of liver fibrosis. When evaluating the elastic properties of circumscribed tissues, the different measurement techniques performed by commercial SWE systems show susceptibility to observational errors, depending upon the ROI and pSWE vs. 2D-SWE technique. The least relative measurement error was observed in situations where the variable ROI is placed within the target using the 2D-SWE technique. For clinical use, the additional assessment of the method is necessary, as the non-standardized conditions yield results that are likely affected by the diameter of the target, the placement of ROI and artifacts caused by surrounding tissue.

Acknowledgment: We thank David Petroff for proof-reading parts of the manuscript.

\section{Conflict of interest: none}

\section{References}

1. Friedrich-Rust M, Vorlaender C, Dietrich CF et al. Evaluation of Strain Elastography for Differentiation of Thyroid Nodules: Results of a Prospective DEGUM Multicenter Study. Ultraschall Med 2016;37:262-270.

2. Friedrich-Rust M, Poynard T, Castera L. Critical comparison of elastography methods to assess chronic liver disease. Nat Rev Gastroenterol Hepatol 2016;13:402-411.

3. Liu B, Zheng Y, Huang G, et al. Breast Lesions: Quantitative Diagnosis Using Ultrasound Shear Wave Elastography - A Systematic Review and Meta-Analysis. Ultrasound Med Biol 2016;42:835-847

4. Cosgrove D, Piscaglia F, Bamber J, et al. EFSUMB guidelines and recommendations on the clinical use of ultrasound elastography. Part 2: Clinical applications. Ultraschall Med 2013;34:238-253. 
5. Zhang ZJ, Ng GY, Lee WC, Fu SN. Changes in morphological and elastic properties of patellar tendon in athletes with unilateral patellar tendinopathy and their relationships with pain and functional disability. PLoS One 2014;9:e108337.

6. Meng W, Xing P, Chen Q, Wu C. Initial experience of acoustic radiation force impulse ultrasound imaging of cervical lymph nodes. Eur J Radiol 2013;82:1788-1792.

7. Gennisson JL, Muller M, Gabor P, et al. Quantification of elasticity changes in the myometrium during labor using Supersonic Shear Imaging: a feasibility study. Ultrasonics 2015;56:183-188.

8. Schiffmann J, Grindei M, Tian Z, et al. Limitations of Elastography Based Prostate Biopsy. J Urol 2016;195:17311736.

9. Londero AP, Schmitz R, Bertozzi S, Driul L, Fruscalzo A. Diagnostic accuracy of cervical elastography in predicting labor induction success: a systematic review and meta-analysis. J Perinat Med 2016;44:167-178.

10. Schröder C, Lock G, Schmidt C, Löning T, Dieckmann KP. Real-Time Elastography and Contrast-Enhanced U1trasonography in the Evaluation of Testicular Masses: A Comparative Prospective Study. Ultrasound Med Biol 2016;42:1807-1815.

11. Albuquerque A. Endoanal ultrasonography in fecal incontinence: Current and future perspectives. World J Gastrointest Endosc 2015;7:575-581.

12. Maksuti E, Widman E, Larsson D, Urban MW, Larsson M, Bjällmark A. Arterial Stiffness Estimation by Shear Wave Elastography: Validation in Phantoms with Mechanical Testing. Ultrasound Med Biol 2016;42:308-321.

13. Cortez CD, Hermitte L, Ramain A, Mesmann C, Lefort T, Pialat JB. Ultrasound shear wave velocity in skeletal muscle: A reproducibility study. Diagn Interv Imaging 2016;97:71-79.

14. Vergari C, Dubois G, Vialle R, et al. Lumbar annulus fibrosus biomechanical characterization in healthy children by ultrasound shear wave elastography. Eur Radiol 2016;26:1213-1217.

15. Zhang YF, Xu HX, He Y, et al. Virtual touch tissue quantification of acoustic radiation force impulse: a new ultrasound elastic imaging in the diagnosis of thyroid nodules. PLoS One 2012; 7:e49094.

16. Bamber J, Cosgrove D, Dietrich CF, et al. EFSUMB guidelines and recommendations on the clinical use of ultrasound elastography. Part 1: Basic principles and technology. U1traschall Med 2013;34:169-184.

17. Chauvet D, Imbault M, Capelle L et al. In Vivo Measurement of Brain Tumor Elasticity Using Intraoperative Shear Wave Elastography. Ultraschall Med 2016;37:584-590.

18. Rouze NC, Wang MH, Palmeri ML, Nightingale KR. Parameters affecting the resolution and accuracy of 2-D quantitative shear wave images. IEEE Trans Ultrason Ferroelectr Freq Control 2012;59:1729-1740.

19. Dillman JR, Chen S, Davenport MS, et al. Superficial ultrasound shear wave speed measurements in soft and hard elasticity phantoms: repeatability and reproducibility using two ultrasound systems. Pediatr Radiol 2015;45:376-385.
20. Carlsen JF, Pedersen MR, Ewertsen C, et al. A comparative study of strain and shear-wave elastography in an elasticity phantom. AJR Am J Roentgenol. 2015;204:W236-W242.

21. Pfeifer L, Goertz RS, Neurath MF, Strobel D, Wildner D. Comparison of Acoustic Radiation Force Impulse (ARFI) Elastography Measurements with the 4C1 Transducer to the 6C1HD Transducer: A Phantom and Patient Study. Ultraschall Med 2016;37:477-481.

22. Shin HJ, Kim MJ, Kim HY, Roh YH, Lee MJ. Comparison of shear wave velocities on ultrasound elastography between different machines, transducers, and acquisition depths: a phantom study. Eur Radiol 2016;26:3361-3367.

23. He YP, Xu HX, Wang D, et al. First experience of comparisons between two different shear wave speed imaging systems in differentiating malignant from benign thyroid nodules. Clin Hemorheol Microcirc 2017;65:349-361.

24. Bende F, Sporea I, Șirli R, et al. Performance of 2D-SWE. GE for predicting different stages of liver fibrosis, using Transient Elastography as the reference method. Med Ultrason 2017;19:143-149.

25. Piscaglia F, Salvatore V, Mulazzani L, Cantisani V, Schiavone C. Ultrasound Shear Wave Elastography for Liver Disease. A Critical Appraisal of the Many Actors on the Stage. Ultraschall Med 2016;37:1-5.

26. Zerhouni MB, Rachedine M, Inventors and Assignees. Ultrasonic calibration material and method. US patent 5,196,343. March 23, 1993.

27. Bland JM, Altman DG. Statistical methods for assessing agreement between two methods of clinical measurement. Lancet 1986;1:307-10.

28. Landers RN. Computing intraclass correlations (ICC) as estimates of interrater reliability in SPSS. The Winnower 2 2015; e143518.81744.

29. Mun HS, Choi SH, Kook SH, Choi Y, Jeong WK, Kim Y. Validation of intra- and interobserver reproducibility of shearwave elastography: Phantom study. Ultrasonics 2013;53:1039-1043.

30. Chang S, Kim MJ, Kim J, Lee MJ. Variability of shear wave velocity using different frequencies in acoustic radiation force impulse (ARFI) elastography: a phantom and normal liver study. Ultraschall Med 2013;34:260-265.

31. Tozaki M, Saito M, Joo C, et al. Ultrasonographic tissue quantification of the breast using acoustic radiation force impulse technology: phantom study and clinical application. Jpn J Radiol 2011;29:598-603.

32. Palmeri ML, Wang MH, Dahl JJ, Frinkley KD, Nightingale KR. Quantifying Hepatic Shear Modulus In Vivo Using Acoustic Radiation Force. Ultrasound Med Biol 2008;34:546-558.

33. Havre RF, Waage JR, Gilja OH, Odegaard S, Nesje LB. Real-Time Elastography: Strain Ratio Measurements Are Influenced by the Position of the Reference Area. Ultraschall Med 2011 Jun 10. doi:10.1055/s-0031-1273247.

34. Fukuhara T, Matsuda E, Fujiwara K, et al. Phantom experiment and clinical utility of quantitative shear wave elastography for differentiating thyroid nodules. Endocr J 2014;61:615-621. 